\title{
A Rare Case of Pancreatitis From Pancreatic Herniation
}

\author{
David Do ${ }^{\mathrm{a}, \mathrm{c}}$, Steven Mudroch ${ }^{\mathrm{a}}$, Patrick Chen ${ }^{\mathrm{a}}$, Rajan Prakash ${ }^{\mathrm{b}}$, \\ Padmini Krishnamurthy ${ }^{\mathrm{b}}$
}

\begin{abstract}
Acute pancreatitis is a commonly encountered condition, and proper workup requires evaluation for underlying causes such as gallstones, alcohol, hypertriglyceridemia, trauma, and medications. We present a case of pancreatitis due to a rare etiology: pancreatic herniation in the context of a type IV hiatal hernia which involves displacement of the stomach with other abdominal organs into the thoracic cavity.
\end{abstract}

Keywords: Pancreatitis; Herniation; Hiatal hernia

\section{Introduction}

Acute pancreatitis is a commonly encountered condition with multiple potential causes including, but not limited to, gallstones, alcohol, hypertriglyceridemia, trauma, toxins, and drugs. We present a rare case of acute pancreatitis, likely from trans-hiatal herniation of the pancreas within a type IV hiatal hernia. Our patient did not have the traditional risk factors for acute pancreatitis. The report of this rare anatomic abnormality contributes to a growing, albeit small, list of cases of acute pancreatitis secondary to pancreatic herniation whereby the pathophysiology may create a challenge for diagnosis and treatment.

\section{Case Report}

A 65-year-old Caucasian male was hospitalized for recurrent abdominal pain, nausea and emesis. He was admitted twice for the same symptoms 1 week apart. His abdominal pain was located the left upper quadrant. The patient was hospitalized 9 months prior, at another facility, for a large para-esophageal hernia and perforation of a gastric ulcer located within the her-

Manuscript submitted March 14, 2018, accepted March 26, 2018

aWright State University, Weber CHE Building, 128 E Apple St, Dayton, OH 45409, USA

bDayton VA Medical Center, 4100 W Third St, Dayton, OH 45428, USA

${ }^{\mathrm{c} C}$ Corresponding Author: David Do, Wright State University, Weber CHE

Building, 128 E Apple St, Dayton, OH 45409, USA.

Email: david.do@wright.edu

doi: https://doi.org/10.14740/jmc3036w nia sac, resulting in mediastinal abscess which was drained surgically. He had a prolonged post-operative recovery following the surgery. In addition, he had a remote history of exploratory laparotomy for a retroperitoneal bleed. He did not have history of alcohol abuse. His past medical history was significant for hypertension and osteoarthritis.

On examination, his vitals showed a heart rate of 106 beats/min, blood pressure of $137 / 85 \mathrm{~mm} \mathrm{Hg}$, temperature of $37.2^{\circ} \mathrm{C}$, and respiratory rate of $20 / \mathrm{min}$. Physical exam demonstrated left-upper quadrant tenderness without guarding or rebound tenderness, with normoactive bowel sounds. Lipase was elevated at 2,687 U/L (reference range: 73 - $383 \mathrm{U} / \mathrm{L}$ ). His lipase with the first episode of abdominal pain had been 1,051 U/L. Hematocrit was 41.2\% (reference range: $42-52 \%$ ), white cell count was $7.1 \mathrm{t} / \mathrm{mm}$ (reference range $4.8-10.8 \mathrm{t} /$ $\mathrm{mm}$ ) and creatinine was $1.0 \mathrm{mg} / \mathrm{dL}$ (references range: $0.5-1.4$ $\mathrm{mg} / \mathrm{dL}$ ). Liver chemistries, serum triglycerides and serum calcium levels were within reference ranges. Ultrasound of the right upper quadrant showed normal common bile duct and no evidence of gallbladder stones or sludge. A computerized tomography (CT) of the abdomen demonstrated a large type IV hiatal hernia through the esophageal hiatus, with near complete intrathoracic displacement of the stomach and a vertically oriented pancreas with herniation of the tail and body of the pancreas into the thorax (Fig. 1). There was no evidence of inflammation of the pancreas. Given his clinical presentation and serum lipase elevation, a diagnosis of mild acute pancreatitis was made and the patient was aggressively hydrated. Upper endoscopy demonstrated a large paraesophageal hernia and two small clean-based ulcers in the body of the stomach. He was prescribed a proton pump inhibitor and his symptoms improved with conservative management. He was discharged 2 days later with plans to be followed up by his surgeon for repair of the hiatal hernia.

\section{Discussion}

Acute pancreatitis is a commonly encountered condition with multiple possible causes including, but not limited to gallstones, alcohol, metabolic derangements, hypertriglyceridemia, drugs, toxins, trauma, and infections. About $20 \%$ of cases of acute pancreatitis are idiopathic [1]. Our patient was diagnosed with acute pancreatitis based on his symptoms and elevated serum lipase. The duration of the paraesophageal hernia was not known as the patient did not have any prior images pertaining to this. The absence of traditional risk factors causing acute 

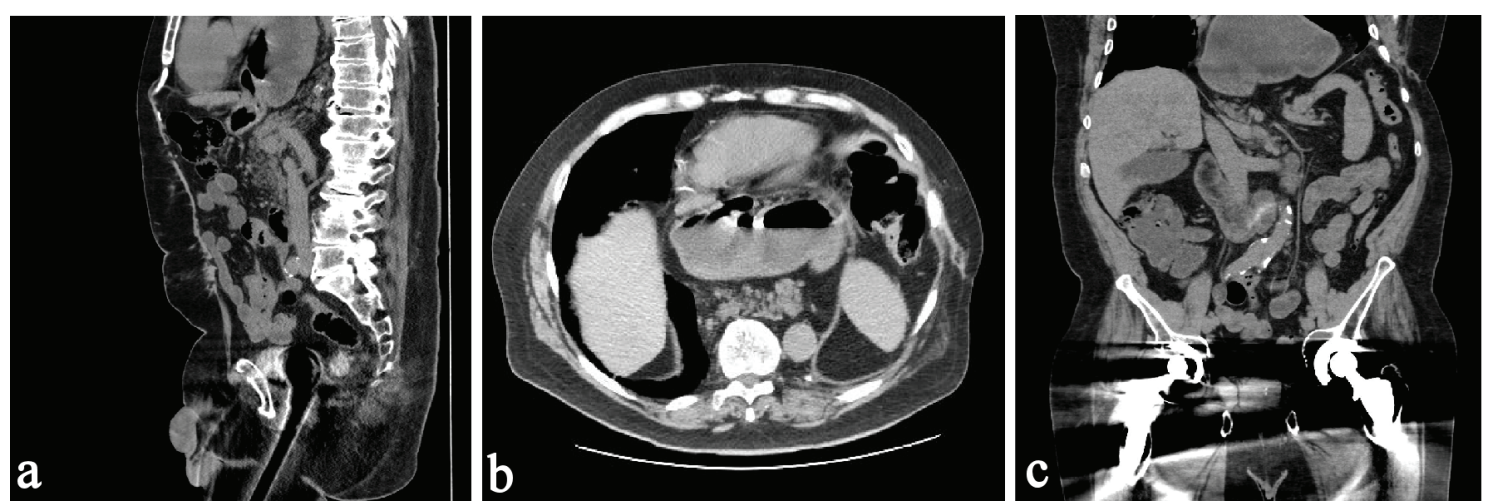

Figure 1. (a) A vertically orientated pancreas is observed with no torsion or malrotation evident. The tail of pancreas, the distal pancreatic body and the majority of stomach is above diaphragm. The head of pancreas and uncinate process is seen below the diaphragm. (b) The stomach and pancreas are seen in the thoracic cavity in this axial image. (c) The stomach and tail of the pancreas are seen in this coronal image.

pancreatitis and the CT findings, led us to believe the etiology of the patient's acute pancreatitis was trans-diaphragmatic herniation of the pancreas.

Hiatal hernias are more common in Western populations, hypothesized to stem from lower fiber intake leading to constipation, straining, and unnaturally raised intra-abdominal pressures [2]. Furthermore, pregnancy, ascites, obesity and trauma may all increase intra-abdominal pressure and can contribute to the development of hiatal hernias [3]. Hiatal hernias are classified into the following categories depending on the extent of herniation and the position of the gastroesophageal junction: sliding hernias (type I), paraesophageal hernias (type II), mixed sliding and paraesophageal hernia (type III), and herniation of additional organs (type IV) $[4,5]$. The latter category is less common, accounting for $5-15 \%$ of cases of hiatal hernias [6]. Type IV hiatal hernias are large and have both a sliding and paraesophageal component with trans-diaphragmatic herniation of intra-abdominal organs including, but not limited to the colon, spleen and pancreas. Symptomatic hiatal hernias, being sliding or paraesophageal, are treated with surgical repair [6]. Some of the gastrointestinal (GI) complications of paraesophageal hernias include gastric volvulus, GI luminal obstruction, incarceration of omentum, or herniated bowel.

Trans-diaphragmatic hernia of pancreas can be asymptomatic and found incidentally on imaging or result in acute pancreatitis. Table $1[3,7-15]$ lists the cases of acute pancreatitis from pancreatic herniation in hiatal hernias from published literature. All cases were diagnosed based on exclusion of other etiologies for acute pancreatitis. The rarity of these cases may be attributable to the fact that the head of the pancreas and the duodenum are anchored by the ligament of Treitz in the retroperitoneum, thereby preventing trans-hiatal herniation of the pancreas $[3,7]$.

The presumed mechanism of pancreatitis due to herniation of the pancreas is thought to be from repetitive trauma of the pancreas and ischemia resulting from intermittent stretching and traction of the vessels supplying the pancreas $[8,9]$. Another possible mechanism includes volvulus formation of the pancreas which prevents normal pancreatic outflow, thereby leading to intraductal hypertension and subsequent inflammation [9].

In conclusion, the etiologies of pancreatitis are diverse, and it is rarely due to pancreatic herniation. Given the rarity of these cases, the exact management of acute pancreatitis secondary to pancreatic herniation is not well established. While

Table 1. Cases of Acute Pancreatitis From Trans-Diaphragmatic Hernia of Pancreas

\begin{tabular}{llllll}
\hline Case & Author & Year & Age/gender & Part of pancreas herniated & Surgery pursued? \\
\hline 1 & Cuschieri et al [11] & 1981 & $19 / \mathrm{M}$ & Tail only & Yes \\
2 & Oliver et al [10] & 1990 & $5 / \mathrm{M}$ & Tail only & Yes \\
3 & Kafka et al [12] & 1994 & N/A & N/A & Yes \\
4 & Chevallier et al [7] & 2001 & $70 / \mathrm{M}$ & Body and tail & No \\
5 & Rozas et al [14] & 2010 & $78 / \mathrm{F}$ & N/A & No \\
6 & Kumar et al [13] & 2013 & $89 / \mathrm{M}$ & Body only & Yes \\
7 & Boyce et al [15] & 2014 & $61 / \mathrm{F}$ & Neck and body & No \\
8 & Lu et al [8] & 2014 & $88 / \mathrm{M}$ & Body and tail & No \\
9 & Patel et al [3] & 2016 & $65 / \mathrm{M}$ & Body and tail & No \\
\hline
\end{tabular}

M: male; F: female; N/A: not applicable. 
several reports have been published of urgent surgical repair, others, including our patient, were managed conservatively [9]. The decision for surgical management depends on the acuity and severity of symptoms, the presence of comorbid conditions, and patient preference. Given serious complications of type IV hiatal hernias, patients known to have this type of hernia need to be followed closely and offered surgical treatment earlier on to prevent serious complications.

\section{Author Contributions}

David Do: primary manuscript writer; Steven Mudroch: editor; Patrick Chen: editor; Rajan Prakash: editor; Padmini Krishnamurthy: editor and guarantor.

\section{Financial Support}

No financial or competing interests to disclose.

\section{Disclosures}

Informed patient consent was obtained for publication of the case report.

\section{Conflict of Interest}

None.

\section{References}

1. Working Party of the British Society of Gastroenterology, Association of Surgeons of Great Britain, Ireland, Pancreatic Society of Great Britain, Ireland, Association of Upper G.I. Surgeons of Great Britain Ireland. UK guidelines for the management of acute pancreatitis. Gut.
2005;54(Suppl 3):iii1-9.

2. Burkitt DP, James PA. Low-residue diets and hiatus hernia. Lancet. 1973;2(7821):128-130.

3. Patel S, Shahzad G, Jawairia M, Subramani K, Viswanathan P, Mustacchia P. Hiatus Hernia: A Rare Cause of Acute Pancreatitis. Case Rep Med. 2016;2016:2531925.

4. Mitiek MO, Andrade RS. Giant hiatal hernia. Ann Thorac Surg. 2010;89(6):S2168-2173.

5. Awais O, Luketich JD. Management of giant paraesophageal hernia. Minerva Chir. 2009;64(2):159-168.

6. Kahrilas PJ. Hiatus hernia causes reflux: fact or fiction? Gullet. 1993;3:21-30,

7. Chevallier P, Peten E, Pellegrino C, Souci J, Motamedi JP, Padovani B. Hiatal hernia with pancreatic volvulus: a rare cause of acute pancreatitis. AJR Am J Roentgenol. 2001;177(2):373-374.

8. Lu LX, Payne M, Theobald CN. Education and imaging. Gastroenterology: Diaphragmatic herniation and pancreatitis. J Gastroenterol Hepatol. 2015;30(4):653.

9. Wang J, Thaker AM, Noor El-Nachef W, Watson RR. Transhiatal herniation of the pancreas: a rare cause of acute pancreatitis. ACG Case Rep J. 2017;4:e66.

10. Oliver MJ, Wilson AR, Kapila L. Acute pancreatitis and gastric volvulus occurring in a congenital diaphragmatic hernia. J Pediatr Surg. 1990;25(12):1240-1241.

11. Cuschieri RJ, Wilson WA. Incarcerated Bochdalek hernia presenting as acute pancreatitis. Br J Surg. 1981;68(9):669.

12. Kafka NJ, Leitman IM, Tromba J. Acute pancreatitis secondary to incarcerated paraesophageal hernia. Surgery. 1994;115(5):653-655.

13. Kumar P, Turp M, Fellows S, Ellis J. Pancreatic herniation: a rare cause of acute pancreatitis? BMJ Case Rep. 2013;2013.

14. Rozas MG, Gonzalez MM. A rare complication of hiatal hernia. Gastroenterology. 2010;139(6):e1-2.

15. Boyce K, Campbell W, Taylor M. Acute pancreatitis secondary to an incarcerated paraoesophageal hernia: a rare cause for a common problem. Clin Med Insights Case Rep. 2014;7:25-27. 